\title{
Evaluation of the possibilities of adapting a constant volume combustion chamber for research on ignition of hypergolic propellants under low and high-pressure conditions
}

In this study, the adaptation possibilities of a constant volume combustion chamber (CVCC) for research on the ignition of hypergolic propellants are presented. The application of hypergolic bipropellants and crucial parameters regarding their ignition behaviour are discussed. The initial studies on ignition delay measurements presented here does not cover the whole range of conditions present in practical systems where hypergolic ignition occurs. In the study, a need for an evaluation of the influence of pressure on the ignition delay was indicated as the reason to conduct research on hypergolic ignition in low and high-pressure environments. Moreover, the study reviews the state-of-the-art experimental methods of investigating the ignition under atmospheric, low and high-pressure conditions, including those utilizing a constant volume combustion chamber. The drop test was pointed out as the most commonly used method; this makes it advantageous in terms of comparing the results with those obtained by other researchers. Therefore, the drop test was selected as a method to be used in a CVCC. The test rig developed here was designed based on a CVCC initially designed for diesel sprays' visualization in high-pressure conditions. All the required modifications, especially the design of the oxidizer dosing unit, are presented in the study.

Key words: hypergolic ignition, hypergolic propellant, ignition delay, drop test, constant volume combustion chamber

\section{Introduction}

Hypergolic propellants are pairs of liquid fuels and oxidizers in which ignition occurs spontaneously upon contact between the two liquids; thereby eliminating the need for a complex ignition system [16]. The reliable restart capability of engines based on hypergolic propellants makes them ideal for spacecraft manoeuvring. An important criterion to evaluate the performance of hypergolic bipropellants is the ignition delay, which is defined as the time from the physical contact of the liquid fuel and the oxidizer to the onset of ignition. A long ignition delay can cause a 'hard-start' problem in which unburnt fuel and oxidizer in the combustion chamber accumulate, then suddenly ignite to generate damaging pressure peaks [5].

Although the existence of hypergolic propellants has been known since 1937 [11], the mechanisms controlling ignition are not yet well understood. The development of hypergolic propellants is highly empirical and current bipropellant hypergolic technology has been in use for more than fifty years. The conventional hypergolic combinations are hydrazine or its derivatives (monomethylhydrazine$\mathrm{MMH}$, or unsymmetrical dimethylhydrazine-UDMH) as a fuel and nitric acid or nitrogen tetroxide (NTO) as an oxidizer. Although these dense, storable propellants give a high performance and were or are used in many systems like the Space Shuttle [7] or Falcon 9 rocket [4], they are highly toxic, difficult to handle and furthermore, hydrazinebased fuels are suspected carcinogens. Accordingly, using the toxic propellants increases the development cost and lead time as well as the risk of disaster for manned space missions. For these reasons, there is strong interest in finding and developing non-toxic hypergolic propellants that have an equal or greater performance than the currently used toxic propellants [6]. Many institutes around the world make efforts to enhance their capabilities in the field of so- called green space propulsion. In numerous cases, a green oxidizer such as high-test peroxide (HTP) was selected and a non-toxic energetic fuel having hypergolicity with this oxidizer was developed $[1,13]$. In this study HTP was also used as an oxidizer.

In order to investigate hypergolic properties of bipropellants, some research methods and procedures have been developed: the drop test and the impingement test. They are focused on investigating ignition delay time. The schematic of the drop test stand is presented in Fig. 1 [9]. The procedure for this experiment is simple: a droplet of an oxidizer is released from a syringe or pipette; it falls down into the pool of a propellant stored in a vessel, e.g. in a small Petri dish; and then the hypergolic ignition is observed.

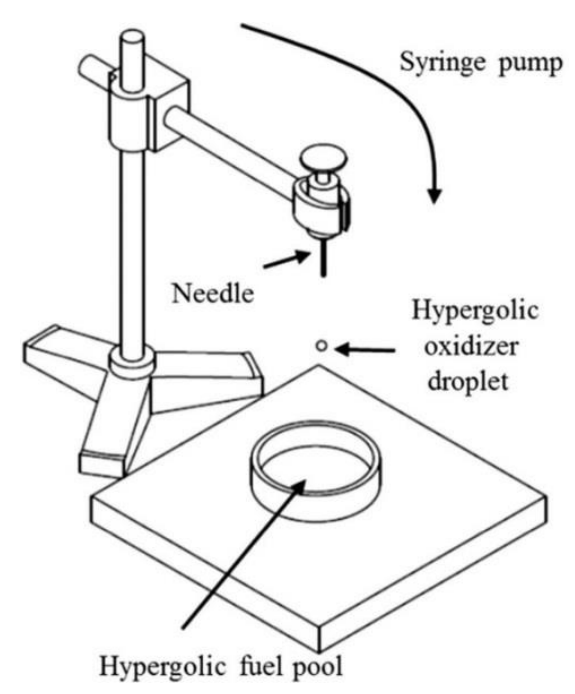

Fig. 1. Schematic drawing of the drop test stand [9] 
The drop test allows the exploration of the variations of ignition delay under two extreme operating conditions: extreme fuel-rich combustion (oxidizer released as a droplet, fuel in the pool); or extreme oxidizer-rich combustion (fuel released as a droplet, oxidizer in the pool). The whole process is recorded by a high-speed camera. In order to more accurately capture the instant of contact of a falling drop with the pool and time of ignition, additional equipment can be utilized, e.g. pressure sensors, photodiodes [18].

The second method of investigating the ignition delay is the impingement test. This test is very similar to the drop test. However, in the impingement test the droplet of the propellant is impinged with a speed of up to $3 \mathrm{~m} / \mathrm{s}$ [8], not dropped as in the drop test. This results in more intensive collision of the fuel and oxidizer; thereby, the ignition delay time is slightly different to that obtained in the drop test.

The drop test stand presented in Fig. 1 enables the conducting of experiments in atmospheric conditions; which is a good starting point to investigate hypergolic properties of bipropellants. Therefore, it can be used as an initial test for the pre-selection of hypergolic propellants, as applies in this study. Here the triglyme with the HTP pair was initially tested in atmospheric conditions in order to evaluate if it is worth further studies.

The drop test in an atmospheric environment is a successful method for initial determination of ignition delay, especially in the early stage of propellant selection. However, it is far away from the conditions in which these propellants are supposed to operate. In combustion chambers of engines used for spacecraft manoeuvring two states of extreme conditions occur-vacuum or very low pressure before the start of the engine and very high pressure after the start of the engine [15]. In terms of the power of the influence of pressure on the ignition delay [2, 14], it is extremely important to measure this parameter under low and highpressure conditions. In order to do this, substantial modifications have to be applied to the standard drop test stand. The drop test stand needs to be isolated from the ambience; this can be done by using a CVCC. These are usually used to conduct optical diagnostics on fuel sprays [10]. High and low-pressure conditions inside the constant volume combustion chamber are achieved by a gas supply system and a vacuum pump. The first is responsible for high pressure conditions and the second for low pressure conditions. When an experiment is supposed to be conducted under high-pressure conditions, air or inert gas from a pressurized tank is supplied to the CVCC. If low-pressure conditions are demanded, then the tank with the gas is closed and vacuum pump removes gases from the CVCC.

There are already a few drop test rigs which utilize the aforementioned solutions [3, 12, 17]. Each of them has different configurations and enables its users to gain some original data from tests. However, there is still room for improvements and fresh ideas in this area. In this study a connection between proven and novel solutions is presented and the possibilities of further development are discussed.

\section{Ignition delay study in atmospheric conditions}

In order to examine the procedures of drop tests and gain initial input for the design assumptions of the high- pressure test rig, a number of experiments under atmospheric conditions $\left(0.1 \mathrm{MPa}, 22{ }^{\circ} \mathrm{C}\right)$ were conducted. As a fuel, triglyme (triethylene glycol dimethyl ether) was selected; and as an oxidizer, high-test peroxide (HTP) was used. In order to react hypergolically with HTP, triglyme requires an addition of, for example, sodium borohydride.

The 'open-air' test rig (Fig. 2) consists of a simple oxidizer dosing system which utilizes a pipette attached to a tripod. Fuel is kept in a watch glass located $200 \mathrm{~mm}$ below the tip of the pipette.

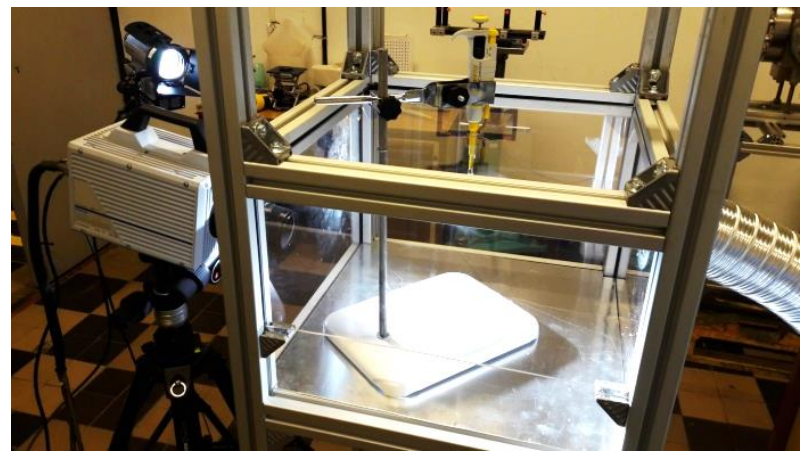

Fig. 2. Drop test rig for measurements in ambient conditions used in the study

As shown in Fig. 2 the operator is separated from the drop test area with polycarbonate sheets. The test volume restricted by polycarbonate walls is continuously scavenged by a ventilation system, which is responsible for removing fumes created during combustion of hypergolic propellants. The falling droplet and ignition are captured using the Photron Fastcam SA-1.1 camera at a frame rate of $10000 \mathrm{fps}$, which requires an additional light source to visualize the droplet and the instant of collision.

\section{Results of tests in atmospheric conditions}

The results of the measured ignition delay obtained in the study are shown in Fig. 3.

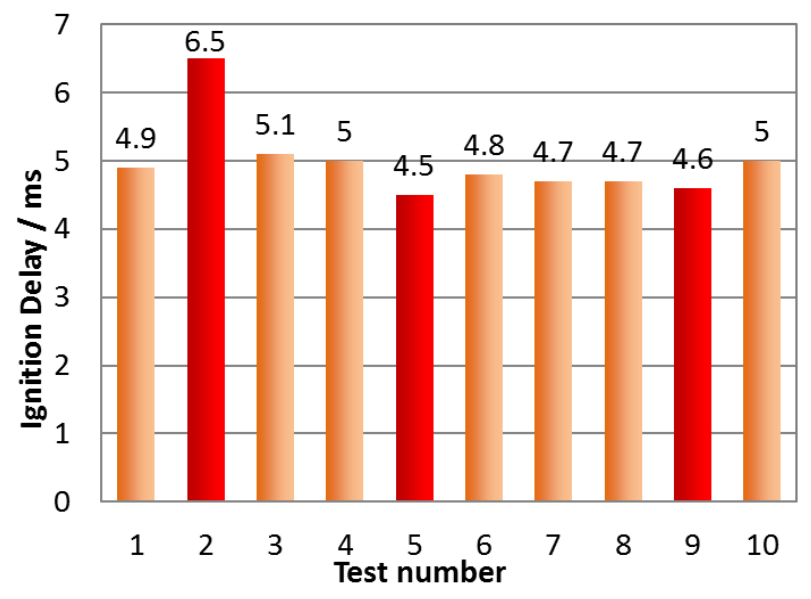

Fig. 3. Measured ignition delay of triglyme-HTP; the cases where the oxidizer droplets did not hit the pool in the centre are marked red

As shown in Fig. 3, the measured ignition delay did not vary between the cases much. The average ignition delay was $4.98 \mathrm{~ms}$ and the standard deviation was $0.57 \mathrm{~ms}$. However, if the cases where the droplets did not hit the fuel pool 
in the centre are excluded (case numbers 2, 5 and 9), then the standard deviation decreases to $0.16 \mathrm{~ms}$. This indicates the importance of a repeatable place of contact between the oxidizer and the fuel. The other issue which can be observed in Fig. 4 is that after releasing a droplet of an oxidizer from a pipette, an additional group of little droplets tend to follow the main droplet. However, these additional droplets did not disturb the ignition delay measurements, because high distance between them and the main droplet caused that they collided with the fuel after the ignition occurred. Nevertheless, this indicates that a more accurate oxidizer dosing system is necessary.
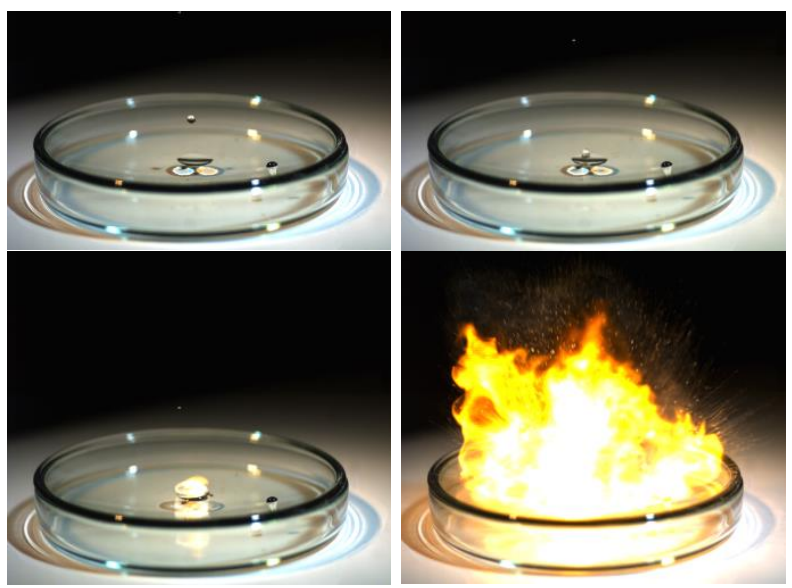

Fig. 4. Drop test of the triglyme-HTP pair; frames obtained at $-5,0,5$ and $10 \mathrm{~ms}$ after the contact of the HTP droplet with the triglyme pool

\section{Drop test stand design}

A constant volume combustion chamber frame (Fig. 5) was the basis for the drop test set-up developed in this study. It was primarily utilized to perform research on fuel sprays [10].

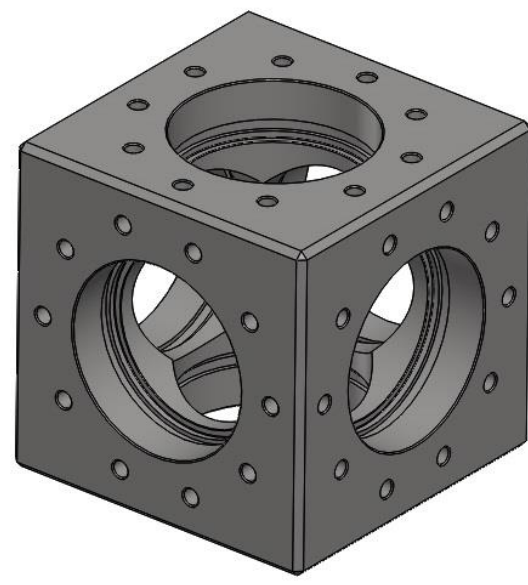

Fig. 5. CVCC frame used as a basis for creating a drop test stand for research in low and high-pressure environments

The major way to adapt the CVCC to drop test experiment was to design the parts attached to the CVCC main frame. Several components attached to the main frame of the vessel were designed:

- Fuel holder
- Oxidizer dosing unit

- Sensors' cover

The fuel holder unit has three main tasks: it closes the CVCC at its bottom; it holds the pillar with a Petri dish filled with fuel; and it is connected to the systems responsible for maintaining low and high pressure inside the CVCC, i.e. the vacuum line and pressurized ambient gas line respectively. Thus, the fuel holder has two ports dedicated to the respective systems. The systems responsible for maintaining low or high-pressure conditions inside the CVCC utilize a vacuum pump and tank with pressurized $\mathrm{N}_{2}$ respectively. When high-pressure conditions are demanded, a valve at the tank is opened and inert gas is supplied to the CVCC. When low-pressure conditions are demanded, the valve at the tank is closed and the vacuum pump is turned on. The vacuum and $\mathrm{N}_{2}$ lines' connections are shown in Fig. 6.

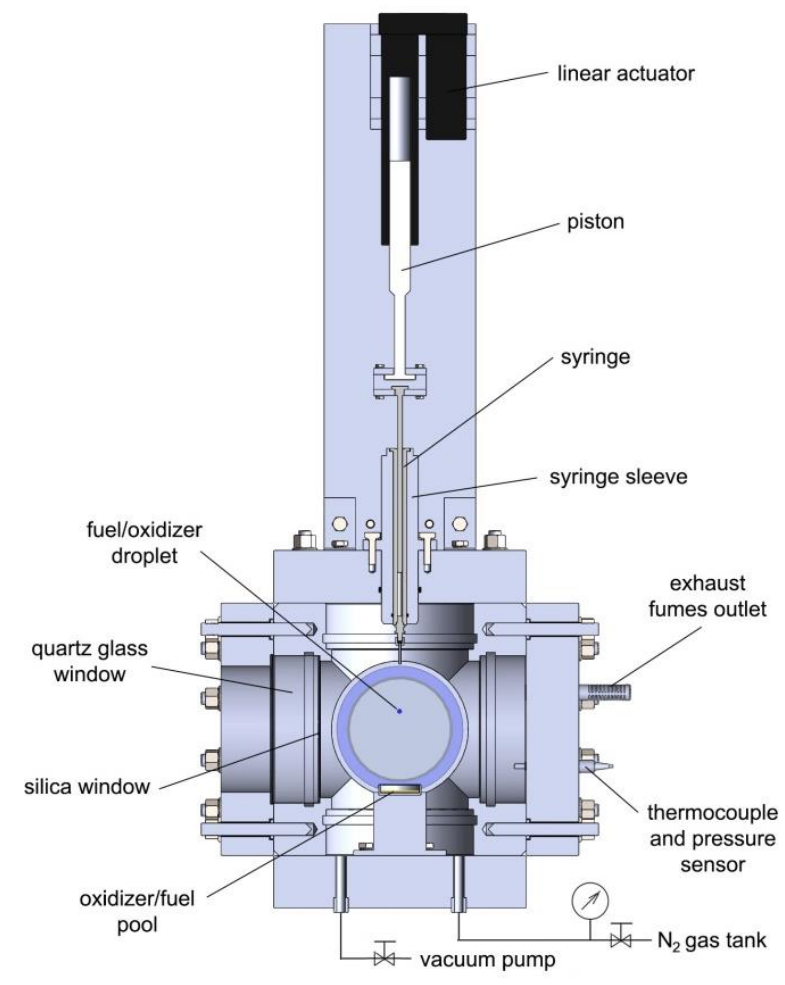

Fig. 6. Schematic diagram of the drop test rig

The oxidizer dosing unit is integrated with an oxidizer supply system which consists of two main elements a syringe of $2 \mathrm{ml}$ capacity and an electric linear actuator. A droplet of an oxidizer of 2.5-3.0 mm diameter is released from the syringe and then falls down into a pool of fuel. The syringe is perched in a stainless steel sleeve which prevents it from movement. The distance from the end of the syringe's needle and the pool of fuel is $120 \mathrm{~mm}$. It was decreased compared to the set-up for the atmospheric tests described in section II. The process of droplet formation is controlled by the electric linear actuator. A part of the actuator referred to as a piston, which moves along a stainless steel guide, pushes the syringe's piston and thereby releases a portion of liquid from the syringe at the end of the needle. The reason why the electric linear actuator was used in the 
study relates to the reliability of the process of droplet formation under high-pressure conditions.

The sensor cover is mounted on the side of the CVCC and it is integrated with three systems:

- pressure measurement system

- temperature measurement system

- exhaust system

The first system includes a pressure transducer and charge amplifier which is connected to a data acquisition unit. The second system consists of a thermocouple, which is connected to the same data acquisition system as the charge amplifier. The third system consists of a gas outlet port connected with a line to release the fumes away from the CVCC after combustion.

In the modified CVCC two quartz windows were designed. The first provides optical access for the camera; while the other allows an externally located light source to illuminate falling droplets. The whole drop test set-up with a high-speed camera is shown in Fig. 7.

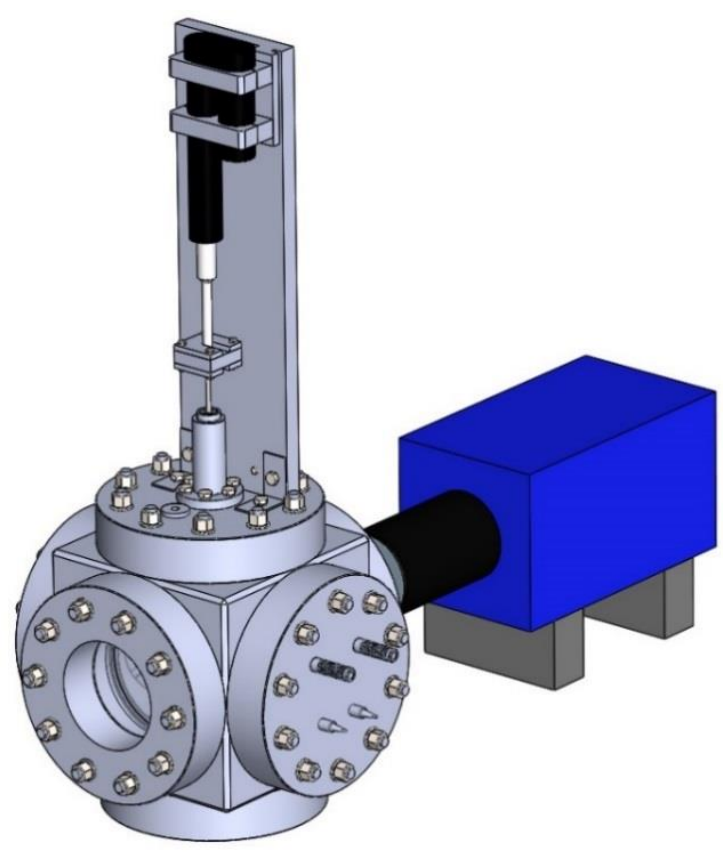

Fig. 7. 3D model of the drop test rig with the camera set-up

\section{Conclusions}

The obtained ignition delay times of the triglyme-HTP pair at atmospheric conditions show that this fuel-oxidizer combination is very promising. The value of $4.98 \mathrm{~ms}$ obtained here is close to the benchmark of hypergolic propellants-monomethylhydrazine-nitrogen tetroxide with an ignition delay time of $3 \mathrm{~ms}$ [16]. However, to fully investigate the potential of this propellant, it is necessary to conduct drop tests not only under atmospheric conditions, but also in a low and high-pressure environment. Therefore, in this study these design solutions intended to adapt a constant volume combustion chamber for research on the ignition of hypergolic propellants under low and high-pressure conditions were proposed.

The initial drop tests in ambient conditions presented in the study revealed also a need for precise droplet dosing, in order to avoid formation of a series of droplets and for an accurate drop test fall into the centre of a fuel pool. These aspects were included in the design process of the high pressure drop test rig.

The test rig development presented here proved that a constant volume vessel initially designed for fuel injection visualization at high-pressure conditions can be utilized to perform drop test studies of hypergolic propellants in low and high-pressure environments.

The number of new systems and modifications were incorporated to the base experimental set-up, e.g. oxidizer dosing system, pressure measurement system, temperature measurement system, exhaust system and systems for creating low and high-pressure conditions in the vessel.

Among these newly designed systems, the most important CVCC upgrade in terms of drop test studies is the oxidizer dosing system. As explained previously, the experiments on the triglyme-HTP propellant conducted in ambient conditions proved that an oxidizer dosing system is of great importance for repeatability of drop tests (formation of droplets and place of contact with the fuel).

All the applied modifications are supposed to ensure high reliability of the experiments; however, further development of the rig is planned, with a number of solutions which are being considered for implementation in the future, e.g. a photodiode which detects the moment of ignition. These, however, will be applied if the first CVCC studies indicate a need for such upgrades.

\section{Acknowledgments}

The paper was prepared as a part of the Hipergol project which is financially supported by the National Centre for Research and Development (NCBiR) under Grant No. 8/2016.

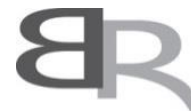

The National Centre for Research and Development

\section{Bibliography}

[1] CONG, Y., ZHANG, T., LI, T. et al. Propulsive performance of a hypergolic $\mathrm{H}_{2} \mathrm{O}_{2}$ /kerosene bipropellant. Journal of Propulsion and Power. 2004, 20(1), 83-86.

[2] CORBETT, A., SEAMANS, T., DAWSON, B. et al. Hypergolic ignition at reduced pressures. Edwards Air Force Base, CA: AFRPL-TR-64-175, 1964.

[3] DAMBACH, E., CHO, K., POURPOINT, T. et al. Ignition of advanced hypergolic propellants. 46th AIAA/ASME/ SAE/ASEE Joint Propulsion Conference \& Exhibit, 2010.
[4] Falcon 9 SpaceX. www.spacex.com/falcon9.

[5] HOLTZMANN, R. Chemical rockets. Marcel Dekker, New York 1969.

[6] HURLBERT, E., APPLEWHITE, J., NGUYEN, T. et al. Nontoxic orbital maneuvering and reaction control systems for reusable spacecraft. Journal of Propulsion and Power. 1998, 14(5), 676-687.

[7] HURLBERT, E., SUN, J., ZHANG, B. Instability phenomena in earth storable bipropellant rocket engines. Progress in Astronautics and Aeronautics. 1995, 169, 122. 
[8] Hypergolic Propellants Laboratory - High Velocity Drop Impact Experiment. engineering.purdue.edu/Hypergol/ high_velocity_impact/index.html.

[9] KANG, H., LEE, E., KWON, S. Suppression of hard start for nontoxic hypergolic thruster using $\mathrm{H}_{2} \mathrm{O}_{2}$ oxidizer. Journal of Propulsion and Power. 2017, 33(5), 1111-1117.

[10] LEWIŃSKA, J., KAPUSTA, Ł.J. Analysis of the microstructure of the fuel spray atomized by marine injector. Combustion Engines. 2017, 169(2), 120-124.

[11] LEY, W. Rockets, missiles, and space travel. Viking Press, New York 1951.

[12] NAtAN, B., Perteghella, V., SOlOMON, Y. Hypergolic ignition of oxidizers and fuels by fuel gelation and suspension of reactive or catalyst particles. 46th AIAA/ ASME/SAE/ASEE Joint Propulsion Conference \& Exhibit 2010.

[13] POURPOINT, T., ANDERSON, W. Hypergolic reaction mechanisms of catalytically promoted fuels with rocket grade hydrogen peroxide. Combustion Science and Technology. 2007, 179(10), 2107-2133.

Maciej Krzesicki, MEng. - Faculty of Power and Aeronautical Engineering, Warsaw University of Technology.

e-mail: Maciej.Krzesicki@ext.itc.pw.edu.pl
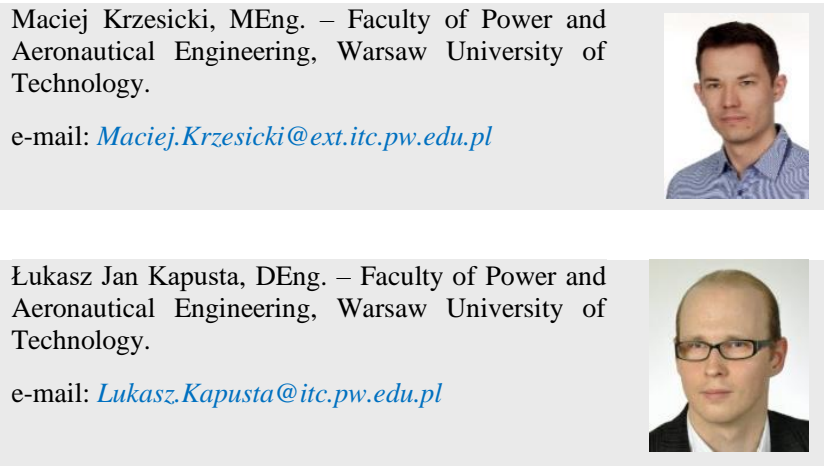

[14] SEAMANS, T., DAWSON, B. Hypergolic ignition at reduced pressures. Edwards Air Force Base, CA: AFRPL-TR64-175, 1967.

[15] SEAMANS, T., VANPEE, M., AGOSTA, V. Development of a fundamental model of hypergolic ignition in spaceambient engines. American Institute of Aeronautics and Astronautics. 1967, 5(9), 1616-1624.

[16] SUTTON, G. History of liquid propellant rocket engines. Alexandria. VA: American Institute of Aeronautics and Astronautics. 2006.

[17] WANG, S., THYNELL, S. Experimental investigation of pressure effect on ignition delay of monomethylhydrazine, 1,1-dimethylhydrazine, tetramethylethylenediamine and 2dimethylaminoethylazide with nitric acid. 8th U. S. National Combustion Meeting 2013.

[18] ZARBO, N., BELAL, H., POURPOINT, T. Effect of water and humidity on hypergolic propellant ignition and combustion. 51st AIAA/SAE/ASEE Joint Propulsion Conference 2015.

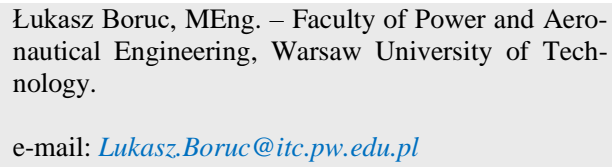

e-mail:Lukasz.Boruc@itc.pw.edu.pl

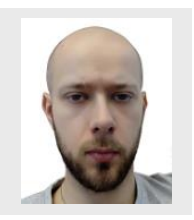

